\title{
Pollutant formation modelling in natural gas SI engines using a level set based flamelet model
}

\author{
R Dahms ${ }^{1 *}$, N Peters ${ }^{1}$, D W Stanton ${ }^{2}$, Z Tan $^{2}$, and J Ewald ${ }^{3}$ \\ ${ }^{1}$ Institute of Combustion Technology, RWTH Aachen, Aachen, Germany \\ ${ }^{2}$ Combustion Research, Cummins Inc., Columbus, Indiana, USA \\ ${ }^{3} \mathrm{FEV}$ Motorentechnik GmbH, Germany
}

The manuscript was accepted after revision for publication on 18 September 2007.

DOI: 10.1243/14680874JER02107

\begin{abstract}
The level set flamelet model for turbulent premixed combustion was recently extended to improve the modelling of the spark ignition process, to incorporate the influence of unsteady flame kernel development on turbulent flame propagation, to embrace flame-wall interaction, and to comprise pollutant prediction models. The formation of nitrogen oxides $\left(\mathrm{NO}_{x}\right)$ is predicted using the extended Zeldovich mechanism. The concentrations of the involved intermediate species are determined using a chemical equilibrium assumption. The formation of engine-out unburnt hydrocarbons (UHCs) and carbon monoxides (COs) is directly linked to incomplete combustion due to flame quenching at the time the flame hits the wall.

In this paper, the new model is used for the analysis of combustion in a homogeneous charged natural gas SI engine operating on lean conditions. The investigated engine operating (PAM) points differ in the applied equivalence ratio, ignition timing, and rate of exhaust gas recirculation. The influence of the load on the peak pressures, peak pressure locations, and pollutant formations is discussed in detail. A good agreement with experimental data was obtained among all engine operating points.
\end{abstract}

Keywords: engine, pollutant modelling, premixed combustion

\section{INTRODUCTION}

According to the US Department of Energy (DOE), over 10 per cent of America's fleet of transit buses and about 20 per cent of newly ordered buses operate on natural gas. Natural gas is one of the cleanest alternative fuels available. For heavy-duty and medium-duty applications, natural gas engines have demonstrated more than 90 per cent reduction of $\mathrm{CO}$ and more than 50 per cent reduction of $\mathrm{NO}_{x}$ relative to commercial diesel engines. For heavy trucks and buses, a modern natural-gas-powered vehicle might reduce, compared with conventional diesel, soot and smog-forming pollution by 75 per cent and 25 per cent respectively. Therefore, there is

\footnotetext{
*Corresponding author: Institute of Combustion Technology, RWTH Aachen, Templergrassen 64, 52056 Aachen, Germany. email:r.dahms@itv.rwth-aachen.de
}

a strong demand to explore and improve natural gas engine concepts. To increase the understanding of the complex interactions between the turbulent flow field and the chemistry, experimental investigations and numerical simulations become an important tool in engine development.

The G-equation concept for premixed and partially premixed turbulent combustion has been successfully applied [1-6] to model bunsen flames, spark ignited engines, and lifted flames. The objective of this work is to develop a new methodology for the modelling of natural gas spark-ignited engines in terms of pollutant prediction. Within this framework, the G-equation flamelet model was extended to incorporate pollutant prediction models for the formation of nitrogen oxides, carbon monoxides $\left(\mathrm{CO}_{\mathrm{s}}\right)$, and unburnt hydrocarbons $\left(\mathrm{UHC}_{\mathrm{s}}\right)$. In this study, five different engine-operating points of a natural-gas-powered engine are presented and compared with experimental data. 
In the first section, the G-equation flamelet model is reviewed. Consequently, the applied pollutant prediction models are derived in detail. In the next section, the experimental and numerical set-up is described and finally, the results are presented and the conclusions and major findings of this study are summarized.

\section{THE G-EQUATION LEVEL SET FLAMELET MODEL FOR TURBULENT PREMIXED COMBUSTION}

Combustion processes in modern spark ignition engines require the modelling of flame propagation commencing with the initial spark, its development to a turbulent flame, and its burn-out when flame quenching occurs. This requires the modelling of the transition process from a laminar flame kernel straight after ignition to a partially developed turbulent flame at the point in time when the flame approaches the walls. In this paper it is exposed that the time between ignition advance and burn-out is not sufficient for the evolution of a fully developed turbulent flame. In typical spark ignition engines the scales of the flame brush thickness and flame propagation speed alter by about one order of magnitude during combustion.

To be able to predict this physical process, it is essential to focus on the high degree of dynamic evolution in terms of modelling. In this paper an algebraic equation for the turbulent flame surface area ratio is presented that comprises unsteady flame development effects on the turbulent burning velocity. Ewald and Peters [3] pointed out that the transport equation of the variance of the mean turbulent flame surface area derived by Peters [1] can be consulted to derive a steady state solution for $\widetilde{G^{\prime \prime}}$ by neglecting the temporal and spatial derivatives. The ratio of the unsteady and steady turbulent flame brush thickness is then introduced into the equation of the turbulent flame surface area ratio to accommodate unsteady flame development effects.

\subsection{Modelling equations for turbulent flame propagation}

The level set approach is used to transport the mean turbulent flame front surface $[\mathbf{7 , 8}]$. It is defined as an iso-surface value that is identified as $G_{0}$ within a scalar field $\tilde{G}$. Outside this iso-surface, the scalar is required to be a signed distance function $|\nabla \tilde{G}|=1$. The kinematic equation valid on the iso-surface reads

$$
\langle\varrho\rangle \frac{\partial \widetilde{G}}{\partial t}+\langle\varrho\rangle \nabla \tilde{u} \widetilde{G}=\left\{\left\langle\varrho S_{\mathrm{T}}\right\rangle-\langle\varrho\rangle \widetilde{\kappa} D_{\mathrm{t}}^{\prime}\right\}|\nabla \widetilde{G}|
$$

To apply a probability density function (PDF) approach to allow for fluctuating corrugated laminar premixed flamelets, their mean deviation from the representative mean turbulent flame front surface needs to be defined. In analogy to the variance equation of a passive scalar, the variance $\widetilde{G^{\prime \prime 2}}$ as the second moment of $\widetilde{G}$ is introduced as follows

$$
\begin{aligned}
& \langle\varrho\rangle \frac{\partial \widetilde{G^{\prime \prime 2}}}{\partial t}+\langle\varrho\rangle \tilde{\boldsymbol{v}}_{\mathrm{f}} \nabla \widetilde{G^{\prime \prime 2}}= \\
& \nabla_{\|}\left(\langle\varrho\rangle D_{t} \nabla_{\|} \widetilde{G^{\prime \prime 2}}\right)+2\langle\varrho\rangle D_{\mathrm{t}}(\nabla \widetilde{G})^{2}-\langle\varrho\rangle \chi_{G}
\end{aligned}
$$

The sink term of the variance $\chi_{G}$ is modelled by a classical approach relating to turbulent dissipation [9]

$$
\chi_{G}=c_{\mathrm{s}} \widetilde{G^{\prime \prime}} \frac{\varepsilon}{k}
$$

The turbulent flame brush thickness can then be related to this variance as follows

$$
\ell_{\mathrm{f}, \mathrm{t}}=\sqrt{\widetilde{G^{\prime \prime 2}}} /|\nabla \tilde{G}|
$$

Such defined first and second moments of $\tilde{G}$ are then used to derive the probability of finding an instantaneous flame front using a Gaussian $\operatorname{PDF} P\left(G ; \widetilde{G}, \widetilde{G^{\prime \prime 2}}\right)$ approach.

The flame surface area ratio is defined to be the proportion of the corrugated and wrinkled flame surface to be modelled and the flame surface provided by the filtered mean turbulent flame front surface equation. Its expression $\widetilde{\sigma}=A_{\mathrm{T}} / \widetilde{A}$ can be related to the turbulent and laminar flame speed ratio $s_{\mathrm{T}} / s_{\mathrm{L}}=\sigma=\sigma_{\mathrm{t}}+1$. The turbulent flame surface area ratio reads

$$
\begin{aligned}
\tilde{\sigma}_{\mathrm{t}} \frac{S_{L}}{v^{\prime}}= & -\frac{a_{4} b_{3}^{2} D a \ell^{*} q+1}{2 b_{1}} \\
& +\sqrt{\left(\frac{a_{4} b_{3}^{2} D a \ell^{*} q+1}{2 b_{1}}\right)^{2}+a_{4} b_{3}^{2} D a \ell^{*} 2}
\end{aligned}
$$

with $q=2 / 3$ [3]. The constants are given in Table 1 .

To incorporate the effect of unsteady flame development on the turbulent flame propagation, the non-dimensional expansion parameter $\ell^{*}$ was 
Table 1 Constants for applied premixed flamelet combustion model

\begin{tabular}{lll}
\hline Symbol & Value & Definition \\
\hline$a_{4}$ & 0.78 & $D_{\mathrm{t}}=a_{4} v^{\prime} \ell$ \\
$b_{1}$ & 2.0 & Experiments $[\mathbf{1 0}]$ \\
$b_{2}$ & 1.78 & {$[\mathbf{1}]$} \\
$b_{3}$ & 1.0 & Experiments $[\mathbf{1 1}]$ \\
$c_{S}$ & 2.0 & {$[\mathbf{7}, \mathbf{1 2}]$} \\
$S c_{t P}$ & 0.7 & \\
$C_{\mu}$ & 0.09 & {$[\mathbf{1 3}]$} \\
\hline
\end{tabular}

introduced. It relates the instantaneous turbulent flame brush thickness to an algebraic thickness assuming homogeneous and steady state conditions with frozen turbulence. This algebraic flame brush thickness can be derived equating production and dissipation in equation (2). This turbulent length scale is then proportional to the integral length scale $\ell_{t}$ and is defined as

$$
\ell_{\mathrm{f}, \mathrm{t}, \mathrm{alg}}=\sqrt{\frac{2 c_{\mu}}{c_{\mathrm{s}} S c_{\mathrm{t}, P}}} \frac{k^{3 / 2}}{\varepsilon}=b_{2} \ell_{\mathrm{t}}
$$

The non-dimensional expansion parameter $\ell^{*}$ follows then to

$$
\ell^{*}=\ell_{\mathrm{f}, \mathrm{t}} / \ell_{\mathrm{f}, \mathrm{t}, \mathrm{alg}}=\ell_{\mathrm{f}, \mathrm{t}} /\left(b_{2} \ell_{\mathrm{t}}\right)
$$

In spark ignition engines, it is assumed that the turbulent eddies may wrinkle and strain the flame but do not perturb the inner reaction zone [14]. Therefore, the laminar burning velocity is not affected by the turbulent flow field. The approximation formula for lean methane-air flames based on an asymptotic analysis from Müller et al. [15], Peters and Williams [16], and Ewald [2] is used for the calculation of the laminar burning velocity taking exhaust gas recirculation (EGR) rates into account.

In order to determine the mean chemical composition of the turbulent flow, the probability density of finding an instantaneous flame front in space and time is determined by presuming the shape of the PDF to be a Gaussian distribution

$$
\widetilde{P}_{\mathrm{b}, \mathrm{ic}}=\frac{1}{m_{\mathrm{ic}}} \int_{V_{\text {ic }}} \bar{\varrho}\left(\widetilde{\xi}_{\boldsymbol{x}}\right) \widetilde{P}_{\mathrm{b}}\left(\widetilde{\xi}_{\boldsymbol{x}}\right) \mathrm{d} V
$$

where

$$
m_{\mathrm{ic}}=\int_{V_{\mathrm{ic}}} \bar{\varrho}\left(\widetilde{\xi}_{\boldsymbol{x}}\right) \mathrm{d} V
$$

The mean density $\bar{\varrho}\left(\widetilde{\xi}_{x}\right)$ is determined from burnt and unburnt states to

$$
\bar{\varrho}\left(\widetilde{\xi}_{\boldsymbol{x}}\right)=\frac{\varrho_{\mathrm{u}} \varrho_{\mathrm{b}}}{\varrho_{\mathrm{u}} \widetilde{P}_{\mathrm{b}}(\widetilde{x})+\varrho_{\mathrm{b}}\left[1-\widetilde{P}_{\mathrm{b}}\left(\widetilde{\xi}_{\boldsymbol{x}}\right)\right]}
$$

The species composition in the unburnt and burnt gas is determined from

$$
\begin{aligned}
\widetilde{Y}_{k}(\boldsymbol{x})= & Y_{k, \mathrm{u}}\left\{1-\widetilde{P}_{\mathrm{b}}\left[\widetilde{G}(\boldsymbol{x}), \widetilde{G^{\prime \prime 2}}(\boldsymbol{x})\right]\right\} \\
& +Y_{k, \mathrm{~b}} \widetilde{P}_{\mathrm{b}}\left[\widetilde{G}(\boldsymbol{x}), \widetilde{G^{\prime \prime 2}}(\boldsymbol{x})\right]
\end{aligned}
$$

\subsubsection{Spark ignition modelling}

The dimensions of typical ignition sparks are smaller than the applied cell sizes of commonly used computational grids. Therefore a subgrid scale model is used to monitor the first stage of spark development.

Straight after ignition the flame thickness of the laminar spark is very thin and the temperature and reactive scalars jump from the unburnt to the burnt state. The burning temperature is obtained by means of an ordinary differential equation at the first stage of combustion as the kernel temperature is higher than the corresponding equilibrium temperature owing to the plasma effects caused by the electrical energy. The energy balance for the spark reads [2]

$$
\dot{Q}_{\mathrm{spk}}-\dot{Q}_{\mathrm{ht}}+\dot{Q}_{\mathrm{chem}}=\frac{\mathrm{d} H}{\mathrm{~d} t}-h_{\mathrm{u}} \dot{m}_{K}-V \frac{\mathrm{d})^{\approx} \mathrm{d} t}{00}
$$

where $\dot{Q}_{\text {spk }}$ denotes the electrical energy transfer from the electrodes to the mixture and $Q_{\mathrm{ht}}$ respects the heat loss of the mixture to the electrodes which is assumed to be $\dot{Q}_{\mathrm{ht}} \approx\left(1-\eta_{e} \mathrm{f}^{0.3} \dot{Q}_{\mathrm{spk}}\right.$. $\dot{Q}_{\mathrm{chem}}$ accounts for the heat release due to combustion which is modelled by $\dot{Q}_{\text {chem }}=\dot{m}_{K}\left(h_{\mathrm{ad}}-h_{\mathrm{u}}\right)$ and $h_{\mathrm{u}}$ represents the specific enthalpy of the unburnt gas mixture which is transported into the spark through the mass stream $\dot{m}_{K}$. The last term is neglected due to an assumed low pressure rise during spark kernel development. After some algebra an ordinary differential equation for the kernel temperature reads on condition of $c_{p} \approx$ const

$$
\frac{\mathrm{d} T_{K}}{\mathrm{~d} t}=-\frac{\dot{m}_{K}}{m_{K}}\left(T_{K}-T_{\mathrm{ad}}\right)+\frac{\dot{Q}_{\mathrm{spk}} \eta_{e f f}}{m_{K} c_{p}}+\frac{1}{\varrho_{\mathrm{b}} c_{p}} \frac{\mathrm{d} p}{\mathrm{~d} t}
$$

The shape of the ignition spark is assumed to be spherical and its centre is convected due to the surrounding flow field. The modelled spark sphere is obtained from 


$$
\begin{aligned}
\vec{x} & =\overrightarrow{x_{0}}+\vec{u} \Delta t \\
r_{K} & =\sqrt[3]{\frac{3 m_{K}}{4 \pi \varrho_{\mathrm{b}}}}
\end{aligned}
$$

The density of the burnt gas is derived from the computed burnt temperature and the flame kernel mass is integrated in time using the following ordinary differential equation

$$
\dot{m}_{K}=4 \pi r_{K}^{2} \varrho_{\mathrm{u}} S_{T, \kappa}
$$

The burning velocity of the mean flame kernel is determined from equation (3) and reads including curvature effects

$$
S_{T, \kappa}=S_{T}-\kappa\left(D_{0}+D_{\mathrm{t}}^{\prime}\right)
$$

Turbulent eddies wrinkle the shape of the kernel which is respected by a non-dimensional transport equation of the variance of the mean flame kernel shape.

$$
\frac{\mathrm{d} \widetilde{G_{\mathrm{spk}}^{\prime 2}}}{\mathrm{~d} t}=2 \hat{D}_{\mathrm{t}, \mathrm{spk}}-c_{\mathrm{s}} \frac{\varepsilon}{\hat{k}_{\mathrm{spk}}} \widetilde{G_{\mathrm{spk}}^{\prime \prime 2}}
$$

The interface among the ignition model and the flow solver is specified using equation (6) which enables the modelling of an inhomogeneous temperature distribution within the flame kernel and allows for heat transfer between the kernel and the unburnt gas.

\subsubsection{Treatment of flame-wall interaction}

The level set-based flamelet model produces approximations of the local turbulent burning velocity and turbulent flame structure of high quality as long as the flame does not touch the wall where flame-wall interactions occur. Influences of these interactions on combustion can be separated into two main groups; the influence of the wall on the chemistry owing to heat loss, and the wall influence on the turbulent length scales. As a general rule, flame-wall interaction reduces the propagation speed of the turbulent flame compared with flames far away from walls.

In this approach, fixed wall temperatures are used to model the heat loss from the gas to the walls. A standard $k-\varepsilon$ approach is applied for the prediction of the Reynold's stresses in conjunction with an algebraic wall function. Both models ensure that the turbulent flame surface area ratio remains reasonable near to walls.
The kinematic equations describing the propagation of the mean turbulent flame front are valid on the iso-surface $G=G_{0}$ only. Upon the turbulent flame hitting the wall, the mean turbulent flame front eventually leaves the domain of integration. In that case propagation velocity can no longer be defined while half of the turbulent flame structure - the flame brush on the burnt gas side - still exists in the combustion chamber. Therefore the flame-wall interaction model ensures that reasonable propagation velocities for the flame brush are formulated and propagated into the integration domain. If necessary, the model locally modifies the isosurface value $G_{0}$ to a new value defined on the burnt gas side of the turbulent flame brush where the kinematic equations are valid from this point onwards.

\section{MODELLING OF POLLUTANT FORMATION}

This section describes the applied strategy to predict engine-out nitrogen oxides, UHCs, and COs for a homogeneous and lean natural gas-air-EGR mixture.

\subsection{Nitrogen oxides}

The extended Zeldovich mechanism, consisting of the classical Zeldovich [17] and the Lavoie mechanism, is used to predict the formation of nitrogen oxides in the engine. Therefore, it is assumed that the thermal $\mathrm{NO}$ is dominant against the $\mathrm{NO}_{2}$, which is neglected in the current approach. The extended Zeldovich mechanism reads

$$
\begin{aligned}
& \mathrm{N}_{2}+\mathrm{O} \rightleftharpoons \mathrm{NO}+\mathrm{N} \\
& \mathrm{N}+\mathrm{O}_{2} \rightleftharpoons \mathrm{NO}+\mathrm{O} \\
& \mathrm{N}+\mathrm{OH} \rightleftharpoons \mathrm{NO}+\mathrm{H}
\end{aligned}
$$

To enable a solution of these three kinetic equations, the concentrations of all species involved have to be calculated. In this approach, an equilibrium solver is applied to estimate the species $\mathrm{N}, \mathrm{H}, \mathrm{O}, \mathrm{OH}$, and $\mathrm{N}_{2}$. NO itself is taken from a three-dimensional transport equation as follows

$$
\frac{\partial\langle\varrho\rangle \widetilde{Y}_{\mathrm{NO}}}{\partial t}+\nabla\langle\varrho\rangle \widetilde{\boldsymbol{u}} \widetilde{Y}_{\mathrm{NO}}=\nabla\left[\left(\frac{\mu}{S c}+\frac{\mu_{\mathrm{t}}}{S c_{\mathrm{t}}}\right) \nabla \widetilde{Y}_{\mathrm{NO}}\right]+\dot{S}_{\mathrm{NO}}
$$


The source term in the transport equation is modelled according to the used Zeldovich mechanism.

The initial species concentration profiles used as start conditions for the fast equilibrium solver are derived from the following global reaction mechanisms

$$
\begin{aligned}
& \mathrm{CH}_{4}+2 \mathrm{O}_{2}+2 \frac{79}{21} \mathrm{~N}_{2}=\mathrm{CO}_{2}+2 \mathrm{H}_{2} \mathrm{O}+2 \frac{79}{21} \mathrm{~N}_{2} \\
& \mathrm{CH}_{4}+\mathrm{O}_{2}+\frac{79}{21} \mathrm{~N}_{2}=\mathrm{CO}+\mathrm{H}_{2}+\mathrm{H}_{2} \mathrm{O}+\frac{79}{21} \mathrm{~N}_{2}
\end{aligned}
$$

\subsection{Unburnt hydrocarbons and carbon monoxides}

The pollutant models are developed as a prediction strategy for lean and homogeneous combustion. Figure 1 shows a schematic representation of the laminar flamelet profile for a lean methane-air mixture obtained from an asymptotic analysis on a four-step reduced mechanism [16].

$$
\begin{aligned}
& \mathrm{I}^{\prime} \quad \mathrm{CH}_{4}+2 \mathrm{H}+\mathrm{H}_{2} \mathrm{O} \rightleftharpoons \mathrm{CO}+4 \mathrm{H}_{2} \\
& \text { II' }^{\prime} \quad \mathrm{CO}+\mathrm{H}_{2} \mathrm{O} \rightleftharpoons \mathrm{CO}_{2}+\mathrm{H}_{2} \\
& \text { III }^{\prime} \quad 2 \mathrm{H}+\mathrm{M} \rightleftharpoons \mathrm{H}_{2}+\mathrm{M} \\
& \text { IV }^{\prime} \quad \mathrm{O}_{2}+3 \mathrm{H}_{2} \rightleftharpoons \mathrm{H}_{2} \mathrm{O}+2 \mathrm{H}
\end{aligned}
$$

\subsubsection{Unburnt hydrocarbons}

According to Fig. 1 the fundamental assumption is derived that in lean combustion all hydrocarbons are totally consumed in the turbulent premixed flame

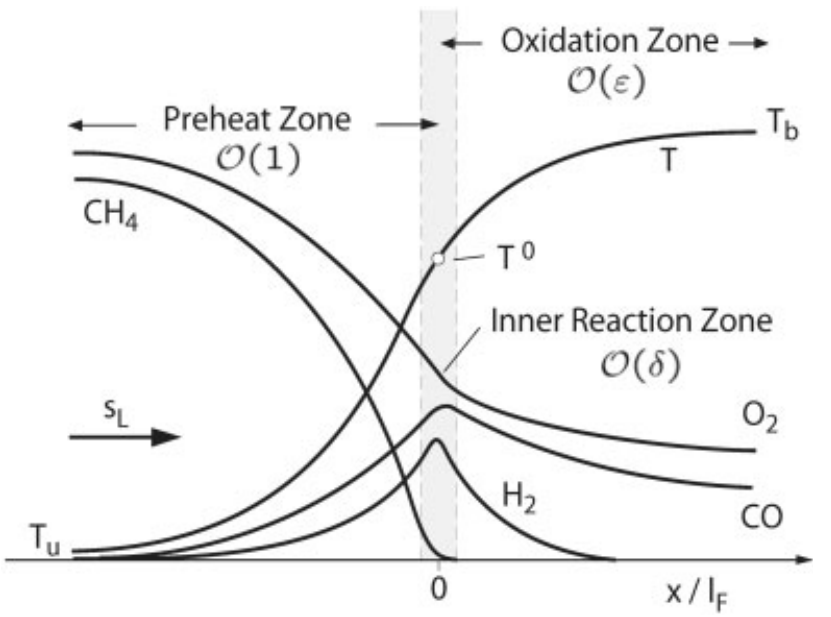

Fig. 1 Illustration of the structure of a premixed methane-air flame front. Therefore, it is assumed that the burnt gas no longer contains any UHCs.

The source of the production of UHCs is found in incomplete combustion when the flame approaches the wall and flame quenching occurs. Then, all hydrocarbons inside the turbulent flame structure remain unburnt in the engine. It is assumed that the characteristic thickness of the turbulent flame right before flame quenching is of about half the turbulent flame brush thickness. The mass of remaining UHCs can then be estimated to

$$
m_{\mathrm{UHC}} \sim \widetilde{\varrho}_{\mathrm{CH}_{4}}\left(t_{\mathrm{FQ}}\right) \widetilde{A}_{\mathrm{T}}\left(t_{\mathrm{FQ}}\right) \frac{1}{2} \ell_{\mathrm{f}, \mathrm{t}}\left(t_{\mathrm{FQ}}\right)
$$

where $\widetilde{\varrho}_{\mathrm{CH}_{4}}$ is introduced as the mean density of methane, $A_{\mathrm{T}}$ as the mean turbulent flame surface area and $\ell_{\mathrm{f}, \mathrm{t}}$ as the turbulent flame brush thickness.

As there are modelling uncertainties, it seems necessary to multiply all results by a constant parameter. Its value is set to $c=0.7$ as the mean of all factors necessary to obtain the provided experimental data. Therefore, the final equation for the prediction of UHCs reads

$$
m_{\mathrm{CH}_{4}, \mathrm{quench}}=0.7 \widetilde{\varrho}\left(t_{\mathrm{FQ}}\right) V_{\text {quench, } 1 / 2}\left(t_{\mathrm{FQ}}\right) \widetilde{Y}_{\mathrm{CH}_{4}}\left(t_{\mathrm{FQ}}\right)
$$

The characteristic flame quenching and flame-wall interaction time $t_{\mathrm{FQ}}$ is identified as the time when the mean turbulent flame surface area reaches its maximum.

\subsubsection{Carbon monoxides}

Although flame quenching is also identified as the leading order effect for $\mathrm{CO}$ production, its prediction model is quite different from that developed for UHCs. Figure 1 illustrates that COs are mainly built in the inner layer of the premixed flame front. Therefore, the model requires that the laminar premixed flamelet structure has to be used for the calculation of the CO production. The shape of the flamelet has to be reconstructed from the mean turbulent flame surface area ratio. The mass of remaining COs can be estimated to

$$
\begin{gathered}
m_{\mathrm{CO}} \sim \underbrace{\left[\widetilde{\sigma}_{\mathrm{t}}\left(t_{\mathrm{FQ}}\right)+1\right] \widetilde{A}_{\mathrm{T}}\left(t_{\mathrm{FQ}}\right)}_{\text {Flamelet shape }} \\
\underbrace{\left[\int\left(\varrho Y_{\mathrm{CO}}\right) \mathrm{d} z\right]\left(t_{\mathrm{FQ}}\right)}_{\text {Flamelet profile }}
\end{gathered}
$$


Table 2 Engine specifications of the Cummins natural gas engine

\begin{tabular}{ll}
\hline Bore $\times$ stroke & $158.75 \times 158.75 \mathrm{~mm}$ \\
Cylinder displacement & $2.7982 \times 10^{-3} \mathrm{~m}^{3}$ \\
Conrod & $289.74 \mathrm{~mm}$ \\
Compression ratio & 11.2 \\
Intake valve closing & $-136^{\circ}$ ATDC \\
Exhaust valve opening & $126^{\circ}$ ATDC \\
TDC clearance & $2 \mathrm{~mm}$ \\
Swirl index & 2.633 \\
Fuel & $\mathrm{CH}_{4}$ (methane) \\
Spark duration & Single spark, $1.5 \mathrm{~ms}$ \\
Spark energy & $60 \mathrm{~J} / \mathrm{s}$ \\
Cylinder head temperature & $573.4 \mathrm{~K}$ \\
Piston head temperature & $516.1 \mathrm{~K}$ \\
Cylinder wall temperature & $451.3 \mathrm{~K}$ \\
\hline
\end{tabular}

using the flame surface area ratio $\widetilde{\sigma}_{\mathrm{t}}$, the mean turbulent flame surface area $A_{\mathrm{T}}$, and the integral expression as the evolution of the density weighted mass fraction of COs in the laminar premixed flamelet.

Owing to modelling uncertainties, a constant parameter is introduced. Its value is set to $c=1.8$ as the mean of all factors necessary to obtain the provided experimental data. Therefore, the final equation for the prediction of COs reads

$$
m_{\mathrm{CO}}=1.8\left[\widetilde{\sigma}_{\mathrm{t}}\left(t_{\mathrm{FQ}}\right)+1\right] \widetilde{A}_{\mathrm{T}}\left(t_{\mathrm{FQ}}\right)\left[\int\left(\varrho Y_{\mathrm{CO}}\right) \mathrm{d} z\right]\left(t_{\mathrm{FQ}}\right)
$$

The time of flame quenching $t_{\mathrm{FQ}}$ is identified as the time when the mean turbulent flame surface area reaches its maximum.

\section{NUMERICAL SIMULATIONS FOR VALIDATION}

At Cummins Inc., Columbus, Indiana, USA, experiments with a natural gas spark ignition engine were carried out. The engine specifications are summarized in Table 2. The topology and the used computational grid of the investigated engine are shown in Fig. 2. It takes the influence of the intake valves and the crevices on the combustion process into account, but neglects the presence of a spark gap and the gasket crevices. Five different engine operating points - PAM points - were investigated, which differ in the applied equivalence ratio, ignition timing, and rate of EGR, see Table 3.

The used flow solver ACFluX solves the incompressible Navier-Stokes equations with a standard $k-$ $\varepsilon$ model to consider a turbulent flow field. The applied initial and boundary conditions for the simulation of the homogeneous charged natural gas spark ignition engine are summarized in Table 4.

The initial turbulent kinetic energy is related to characteristic turbulent length and timescales. From dimensional analysis, it follows that $k \sim\left(L_{\mathrm{c}} / t_{\mathrm{c}}\right)^{2}$. The expression $k=0.3$ (stroke $\mathrm{r} / \mathrm{min} / 30.0)^{2}$ was applied successfully. The initial turbulent eddy dissipation rate is computed due to the turbulent kinetic energy and a characteristic length scale. In this approach, the distance to the walls is used to model the initial turbulent length scale distribution inside the engine according to $\varepsilon=0.15\left(k^{1.5} / L_{\mathrm{c}}\right)$
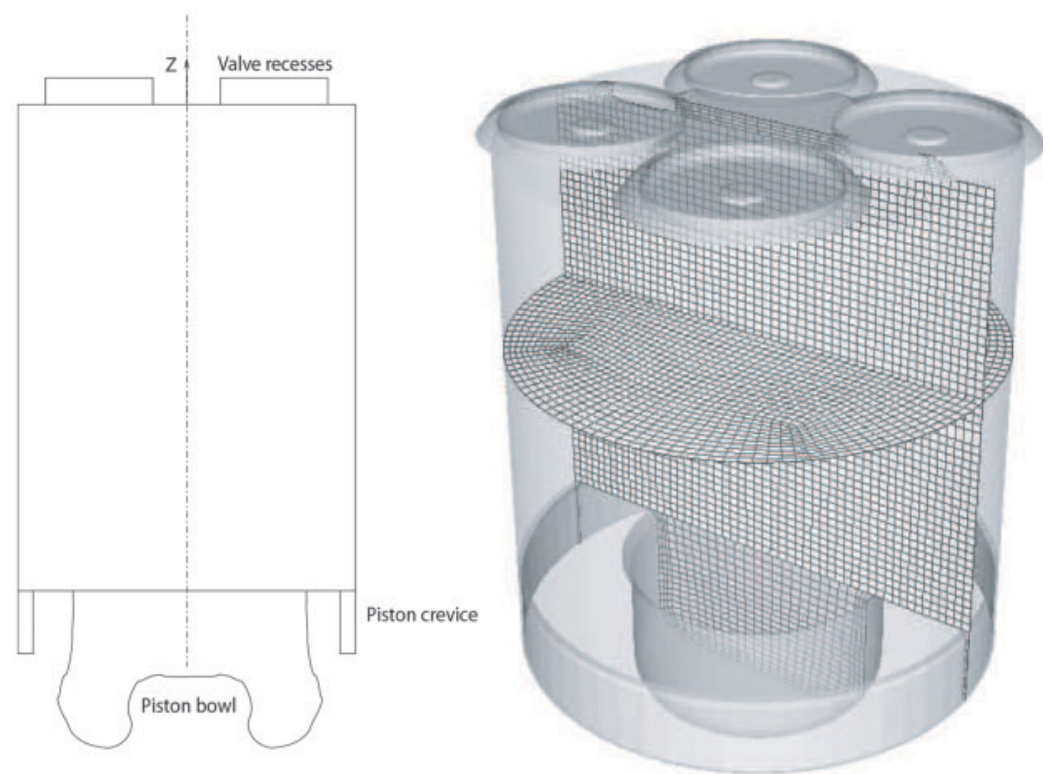

Fig. 2 Topology and unstructured computational grid of the engine geometry. At inlet valve closure (IVC) and top dead centre (TDC) the grid comprises 152080 and 21880 grid cells respectively 
Table 3 Engine specifications of PAMs

\begin{tabular}{|c|c|c|c|c|c|}
\hline & \multicolumn{5}{|c|}{ PAM point } \\
\hline & 902 & 904 & 906 & 908 & 910 \\
\hline Manifold pressure (kPa) & 186.5 & 209.6 & 212.7 & 231.8 & 273.5 \\
\hline Equivalence ratio & 0.66 & 0.76 & 0.84 & 0.93 & 1.0 \\
\hline EGR (\%) & 9.4 & 20.5 & 23.2 & 24.4 & 25.8 \\
\hline Engine speed $(\mathrm{r} / \mathrm{min}$ & 1200 & 1201 & 1202 & 1203 & 1204 \\
\hline Ignition advance ( ${ }^{\circ}$ ATDC) & -19.0 & -20.0 & -21.0 & -22.0 & -23.0 \\
\hline
\end{tabular}

Table 4 Computational initial and boundary conditions

\begin{tabular}{|c|c|c|c|c|c|}
\hline & \multicolumn{5}{|c|}{ PAM point } \\
\hline & 902 & 904 & 906 & 908 & 910 \\
\hline$Y_{\text {Res }}$ & 0.06926 & 0.1781 & 0.2216 & 0.2412 & 0.2546 \\
\hline$Y_{\text {Fuel }}$ & 0.03434 & 0.03461 & 0.03613 & 0.03868 & 0.04161 \\
\hline$Y_{\text {Air }}$ & 0.8964 & 0.7873 & 0.7423 & 0.7201 & 0.7038 \\
\hline$Y_{\text {Prod }}$ & 0.0 & 0.0 & 0.0 & 0.0 & 0.0 \\
\hline Air-fuel ratio $\left.\right|_{\text {st }}$ & 18.61 & 21.14 & 22.47 & 23.16 & 23.70 \\
\hline Initial spark radius (mm) & 2.0 & 2.0 & 2.0 & 2.0 & 2.0 \\
\hline
\end{tabular}

\section{RESULTS AND DISCUSSION}

In this chapter, the results of the calculations of the G-equation and pollutant models for all PAMs are presented and compared with experimental data provided by Cummins, Inc.

\subsection{Level set flamelet model}

This section presents the results of the calculated pressure traces, burnt fuel mass fractions, and apparent heat release rates in comparison with the experimental data provided by Cummins, Inc. for all PAMs. In Fig. 3, the engine operating conditions are classified using the regime diagram from Peters [1] for turbulent premixed combustion. The quantities are averaged over the mean turbulent flame front surface for the corresponding crank angle position.
The combustion takes place either in the thin reaction zone or in the corrugated flamelet regime. Therefore, the second Karlovitz number $K a_{\delta}=\ell_{\delta} / \eta$ relating the inner layer thickness $\ell_{\delta}$ to the Kolmogorov length scale $\eta$ remains less than unity. The Kolmogorov eddies cannot perturb the inner reaction zone and the chemical timescale remains unaffected by turbulence. This confirms that the scale separation between the chemical kinetics for the destination of the laminar burning velocity and the turbulence for the computation of the turbulent flame surface area ratio $\sigma_{\mathrm{t}}$ in the applied G-equation model is valid. After the first stage of ignition where the flame kernel is overall laminar up to the point in time of peak pressure location, the combustion takes place in the thin reaction zone regime. There, the increase of the turbulent burning velocity with the turbulence intensity approaches the square root

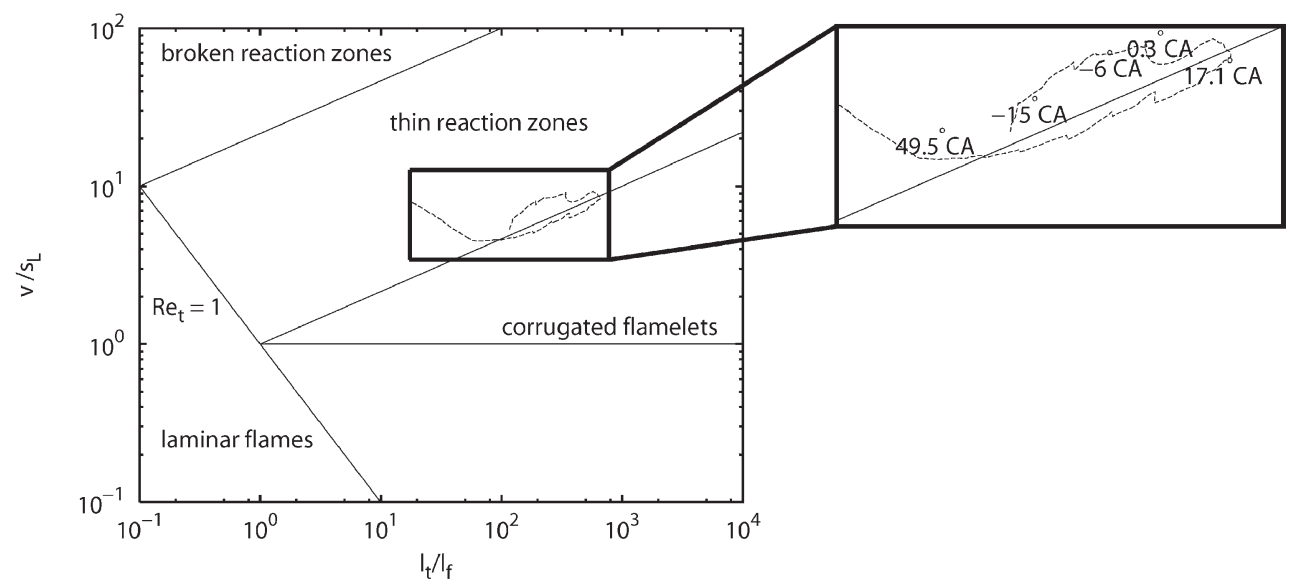

Fig. 3 Location of PAM point 908 in the regime diagram 

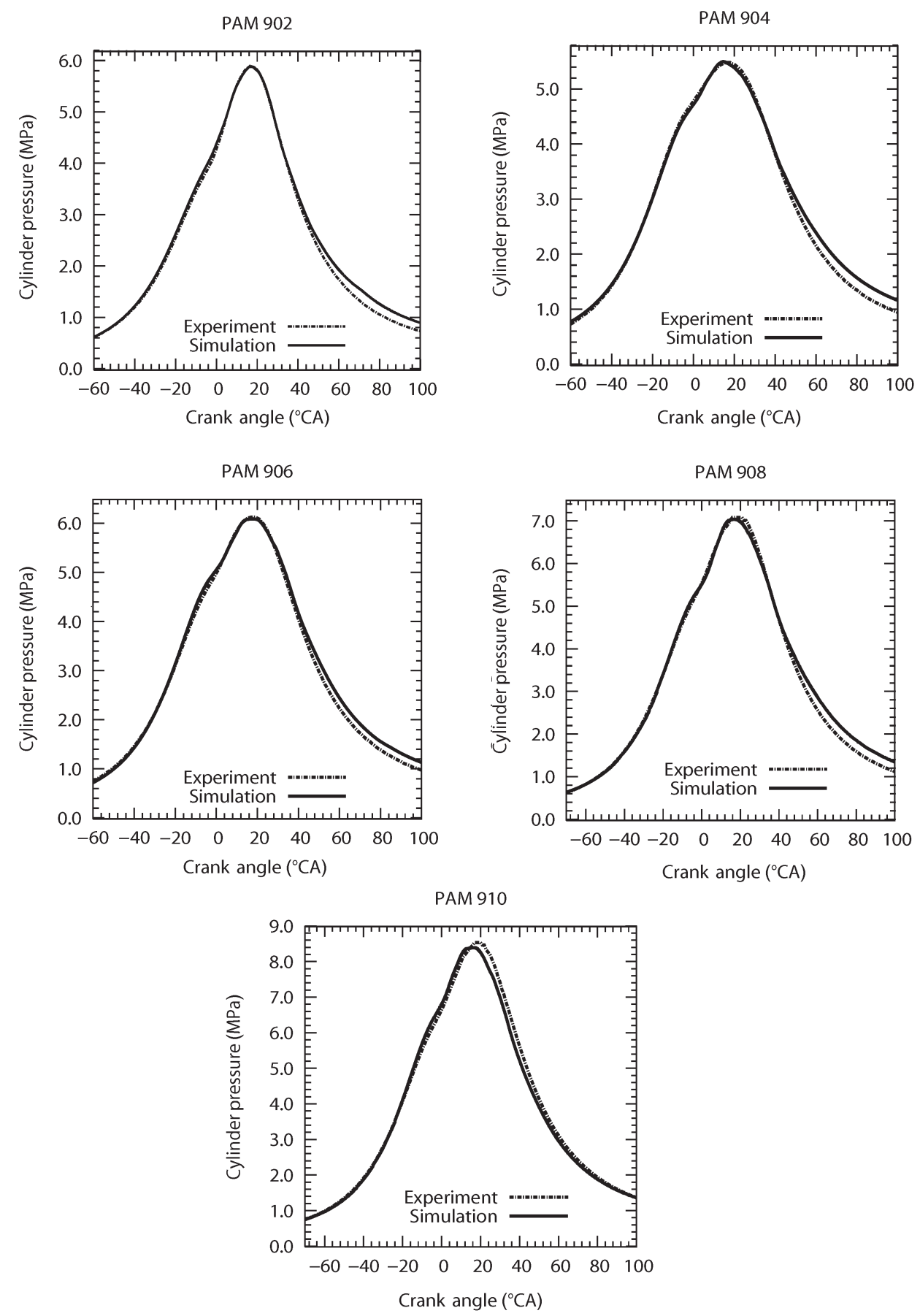

Fig. 4 Simulated/measured cylinder pressures for all PAMs

dependence founded by Damköhler [18]

$$
\frac{S_{\mathrm{T}}}{S_{\mathrm{L}}} \sim\left(\frac{D_{\mathrm{t}}}{D}\right)^{1 / 2} \sim\left(\frac{v^{\prime} \ell}{S_{\mathrm{L}} l_{\mathrm{F}}}\right)^{1 / 2}
$$

The pressure traces are presented in Fig. 4 and they are in overall good agreement with the experimental data. The combustion advance from ignition timing up to the 70 per cent burn point including the peak pressure and peak pressure locations are even excellently caught for all PAM points; see Fig. 5 and Table 5 for further illustration. This underlines that the simulation enables turbulent flame propagation to be predicted successfully over a wide variety of thermodynamic conditions. However, in the last phase of combustion, the burning rate slows down and the applied model failed to predict correctly the 90 per cent burn point for all investigated PAMs; see Table 5 for reference. The 

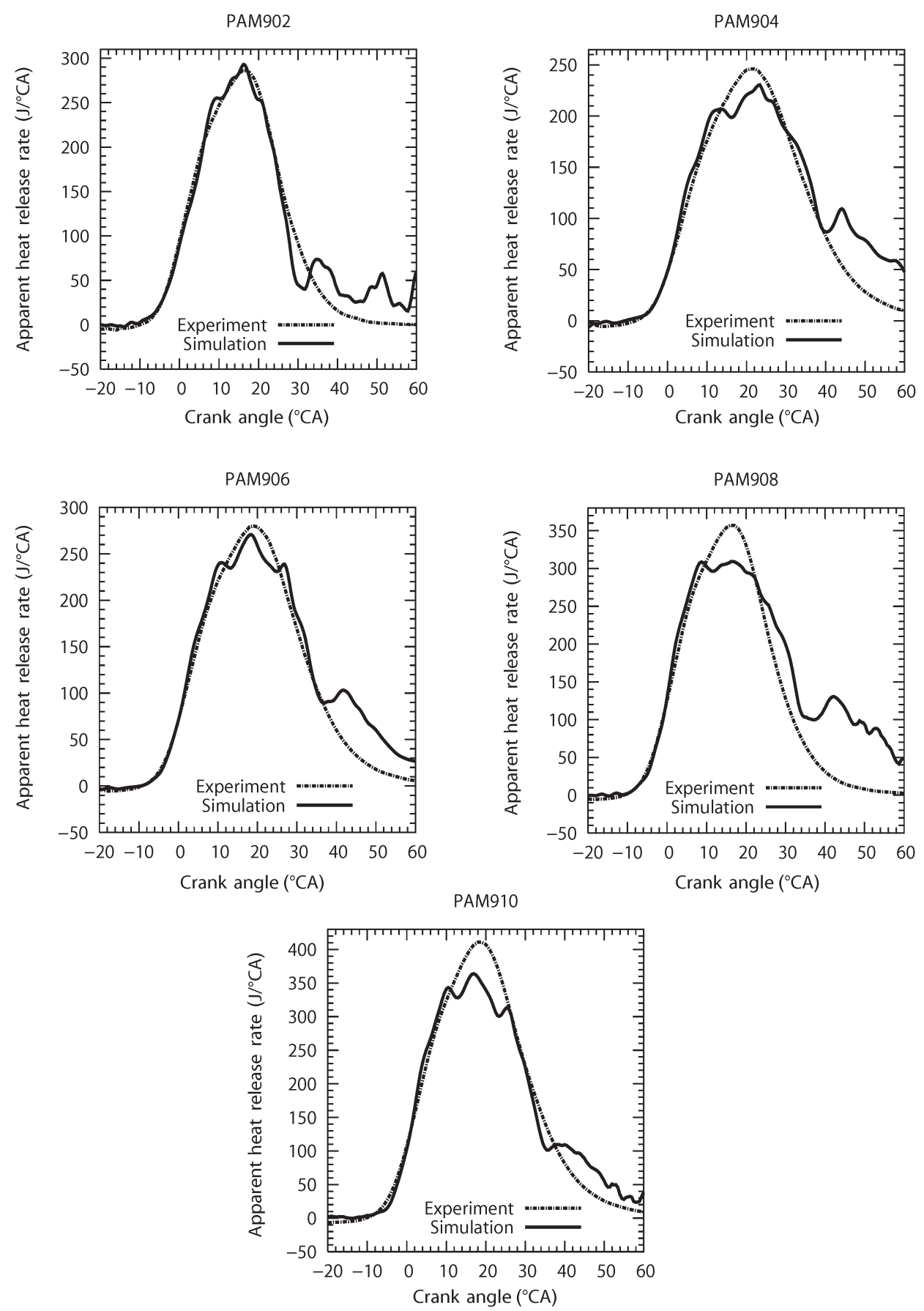

Fig. 5 Simulated/measured apparent heat release rates for all PAMs

apparent heat release rates show a great number of kinks in that last phase of combustion.

In Fig. 6 the mean turbulent flame front is shown nearby the 2 per cent, 10 per cent, 50 per cent and 90 per cent burn points. It can be seen that at about $10^{\circ}$ after top dead centre (ATDC) the flame enters the squish region.

\subsection{Pollutant prediction models}

In the following section, the results of the calculations of the pollutant prediction models for nitrogen oxides, UHCs, and COs are presented. All pollutant model results are in good agreement with the experimental data provided by Cummins, Inc. 
Table 5 Simulated peak pressures, peak pressure locations, and burn points compared with experimental data

\begin{tabular}{|c|c|c|c|c|c|}
\hline & \multicolumn{5}{|c|}{ PAM point } \\
\hline & 902 & 904 & 906 & 908 & 910 \\
\hline \multicolumn{6}{|l|}{ Peak pressure } \\
\hline Experiment (MPa) & 5.90 & 5.50 & 6.14 & 7.10 & 8.55 \\
\hline Simulation (MPa) & 5.89 & 5.52 & 6.10 & 7.07 & 8.43 \\
\hline \multicolumn{6}{|l|}{ Peak pressure location } \\
\hline Experiment ( ${ }^{\circ}$ ATDC) & 17.0 & 16.6 & 18.0 & 18.2 & 18.5 \\
\hline Simulation ('ATDC) & 17.2 & 15.0 & 17.7 & 17.1 & 16.6 \\
\hline \multicolumn{6}{|l|}{ Burn point $2 \%$} \\
\hline Experiment $\left({ }^{\circ} \mathrm{ATDC}\right)$ & -2.15 & 0.40 & -0.85 & -2.74 & -1.93 \\
\hline Simulation ( ${ }^{\circ}$ ATDC) & -3.50 & -1.90 & -1.80 & -6.15 & -2.74 \\
\hline \multicolumn{6}{|l|}{ Burn point $10 \%$} \\
\hline Experiment $\left({ }^{\circ}\right.$ ATDC) & 3.20 & 6.85 & 5.18 & 2.80 & 4.24 \\
\hline Simulation ( $\left.{ }^{\circ} \mathrm{ATDC}\right)$ & 2.90 & 5.40 & 5.00 & 0.30 & 3.80 \\
\hline \multicolumn{6}{|l|}{ Burn point $50 \%$} \\
\hline Experiment $\left({ }^{\circ}\right.$ ATDC $)$ & 15.72 & 22.60 & 19.74 & 15.89 & 18.23 \\
\hline Simulation ( ${ }^{\circ}$ ATDC) & 16.28 & 23.40 & 21.20 & 17.10 & 19.80 \\
\hline \multicolumn{6}{|l|}{ Burn point $90 \%$} \\
\hline Experiment ( ${ }^{\circ}$ ATDC) & 31.80 & 42.51 & 37.60 & 30.46 & 34.86 \\
\hline Simulation ('ATDC) & 34.00 & 53.90 & 48.30 & 49.50 & 48.10 \\
\hline
\end{tabular}
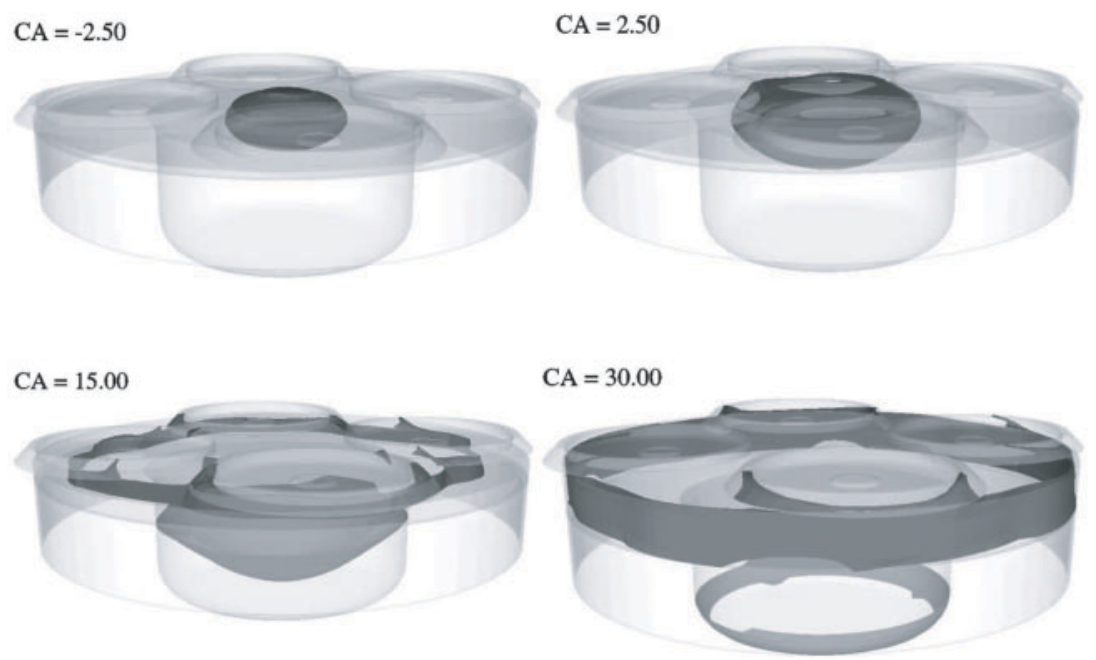

Fig. 6 Visualization of the mean turbulent flame front nearby the 2 per cent, 10 per cent, 50 per cent, and 90 per cent burn points

\subsubsection{Nitrogen oxides}

From the $\mathrm{NO}_{x}$ evolution traces in Figs 7 and 8, it can be seen that $\mathrm{NO}_{x}$ is only built, because the implemented model describes the physical phenomenon of $\mathrm{NO}_{x}$ formation as purely cumulative. This is in line with the applied Zeldovich mechanism, because it just describes a $\mathrm{NO}_{x}$ formation path. The creation of $\mathrm{NO}_{x}$ initiates after the 10 per cent burn point where the mean cylinder temperature is above approximately $1000 \mathrm{~K}$. This presents the strong temperature dependency of the Zeldovich mechanism owing to the high activation energy of $319 \mathrm{~kJ} /$ mole of reaction (R1). The $\mathrm{NO}_{x}$ formation is only stopped when the burning rate drops below a certain level and the production of involved intermediates starts to decrease. For illustration, the evolution of the intermediate specie $\mathrm{OH}$ is also plotted on top of the $\mathrm{NO}_{x}$ traces in Fig. 8 .

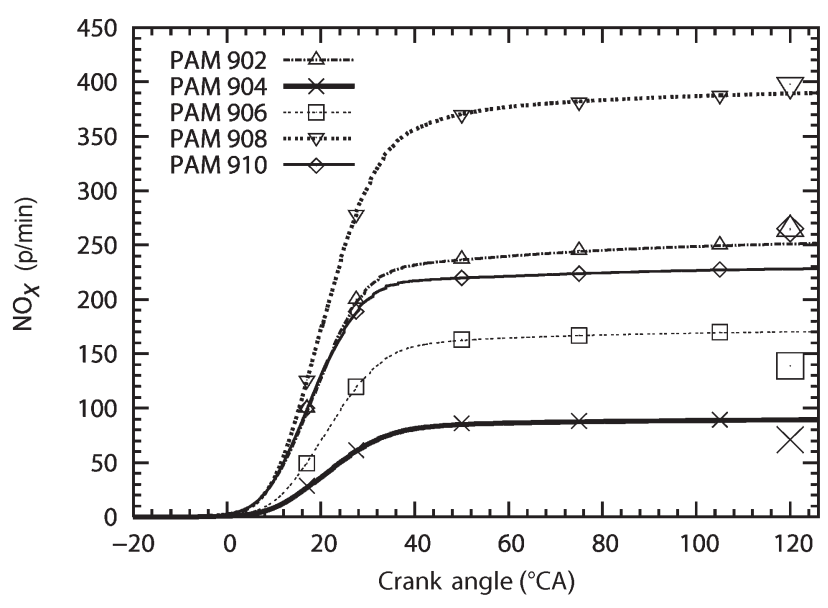

Fig. 7 Simulated/measured $\mathrm{NO}_{x}$ mole fractions for all PAMs (thick symbols indicate measurement at exhaust valve opening (EVO) 

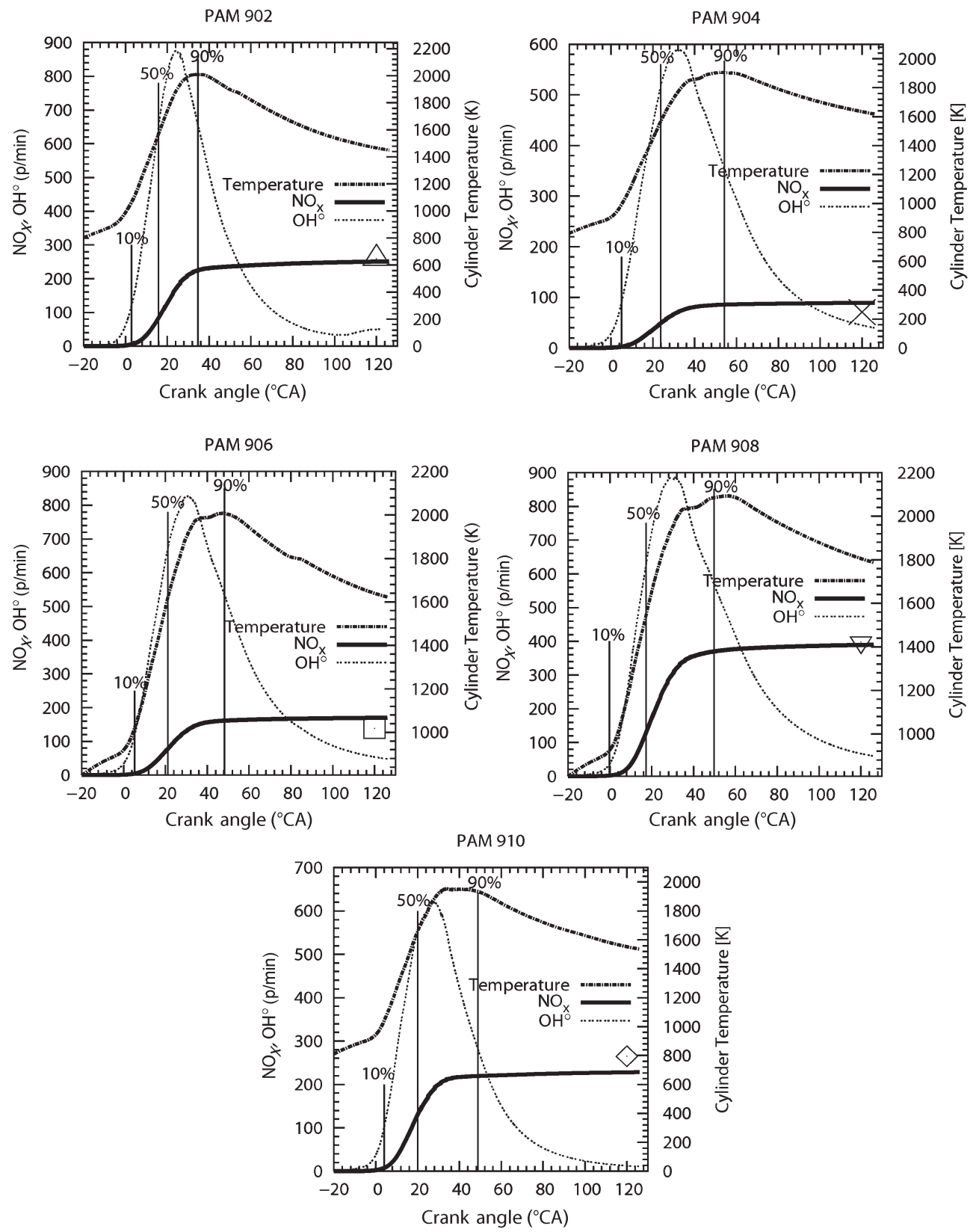

Fig. 8 Evolution of $\mathrm{NO}_{x}, \mathrm{OH}$, and mean cylinder temperature for all PAMs (symbols indicate measurement at EVO)

Another formation path for NO, the Fenimore mechanism [19], is not taken into account. That may explain the underproduction of the $\mathrm{NO}_{x}$. The contribution of prompt NO increases with increasing EGR rate [20] and has a special meaning for moderately rich mixtures $[\mathbf{2 1}]$. Nevertheless, the Zeldovich mechanism with all intermediate species taken from an equilibrium solver is sufficient to predict $\mathrm{NO}_{x}$ within an acceptable reliability, see Table 6.

\subsubsection{Unburnt hydrocarbons}

In Figs 9 and 10, the evolutions of all model quantities used for the prediction of UHC - the turbulent flame surface area and the mean turbulent flame brush thickness - are plotted among all PAMs.

The final results from the UHC pollutant prediction model are in good agreement with the experimentally measured data provided by Cummins, Inc. 
Table 6 Simulated and measured pollutant formations

\begin{tabular}{lccccc}
\hline & \multicolumn{5}{c}{ PAM point } \\
\cline { 2 - 6 } & 902 & 904 & 906 & 908 & 910 \\
\hline Unburnt hydrocarbons & 2451 & 2850 & 2516 & 2154 & 2651 \\
$\quad$ Experiment (ppm) & 2539 & 2650 & 2526 & 2271 & 2614 \\
$\quad$ Simulation (ppm) & & & 562 & 639 & 683 \\
Carbon monoxides & 546 & 590 & 500 & 652 & 666 \\
$\quad$ Experiment (ppm) & 487 & 71 & 139 & 398 & 265 \\
$\quad$ Simulation (ppm) & 264 & 92 & 168 & 390 & 229 \\
$\quad$ Nitrogen oxides & 252 & & & & \\
$\quad$ Experiment (ppm) & & & & & \\
\hline
\end{tabular}

The graph in Fig. 11 shows that the simulation enables the trend of the production of UHCs to be followed among all PAMs.

Following the model equation, the results depend on the combustion chamber geometry, the charge, the flow field, and the ignition timing. In this study, the combustion chamber geometry is all the same among the PAMs. The mean flow field and turbulence level will also remain similar as the engine speed was nearly held constant. Therefore, the production of UHCs does not change drastically among all PAM points.

\subsubsection{Carbon monoxide}

In Figs 9 and 12, the evolutions of the mean turbulent flame surface area and the mean turbulent flame surface area ratio used for the geometrical reconstruction of the laminar flamelets are plotted among all PAMs. In Fig. 13, the evolution of the different profiles of the one-dimensional laminar flamelets among all PAMs computed on conditions

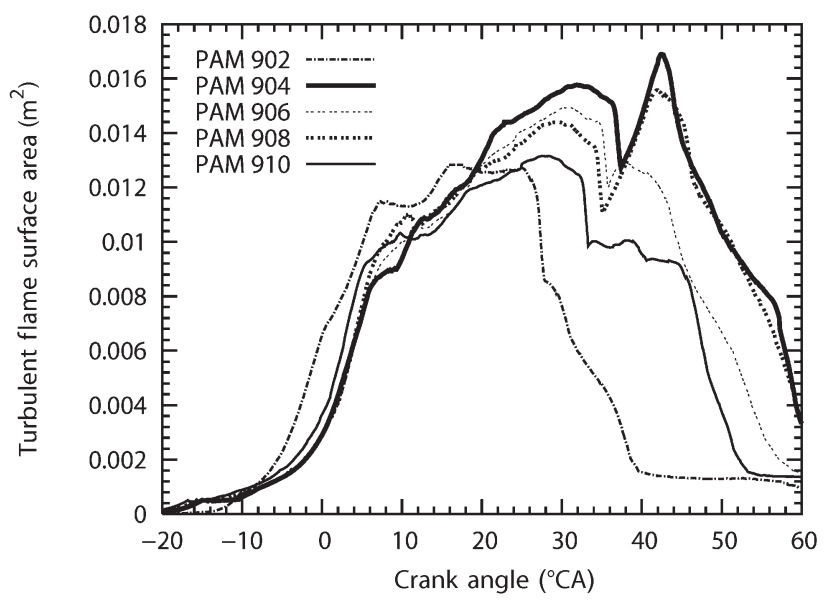

Fig. 9 Mean turbulent flame surface area $\tilde{A}_{\mathrm{T}}$. Quantities are averaged over the values of the mean turbulent flame front surface occurring at points in time when flame quenching occurs is shown.

The final results of the CO pollutant prediction model are in good agreement with the experimentally measured data. The model is able to follow the trend of the production of COs among all operating points, compare Fig. 14.

\subsection{Summary and conclusion}

Within the framework of the G-equation, an approach was presented to account for unsteady flame development and flame-wall interaction effects on the turbulent burning velocity. The modelling of nitrogen oxides based on a combination of an equilibrium solver and the extended Zeldovich mechanism was shown as well as phenomenological models to capture the formation of $\mathrm{CO}$ and $\mathrm{UHC}$ emissions.

The new approach was applied to simulate a homogeneous operating natural gas spark ignition engine. Five different PAMs were considered.

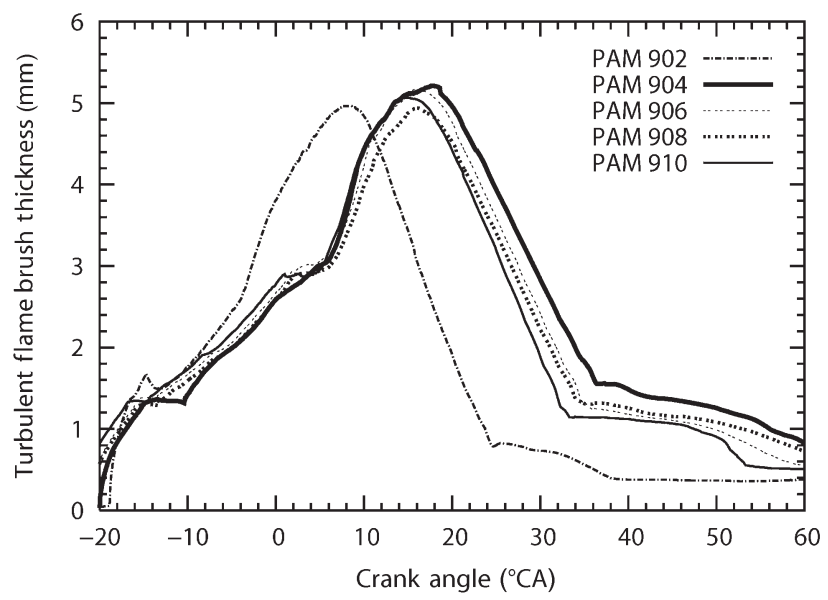

Fig. 10 Turbulent flame brush thickness $\ell_{\mathrm{f}, \mathrm{t}}$. Quantities are averaged over the values of the mean turbulent flame front surface 


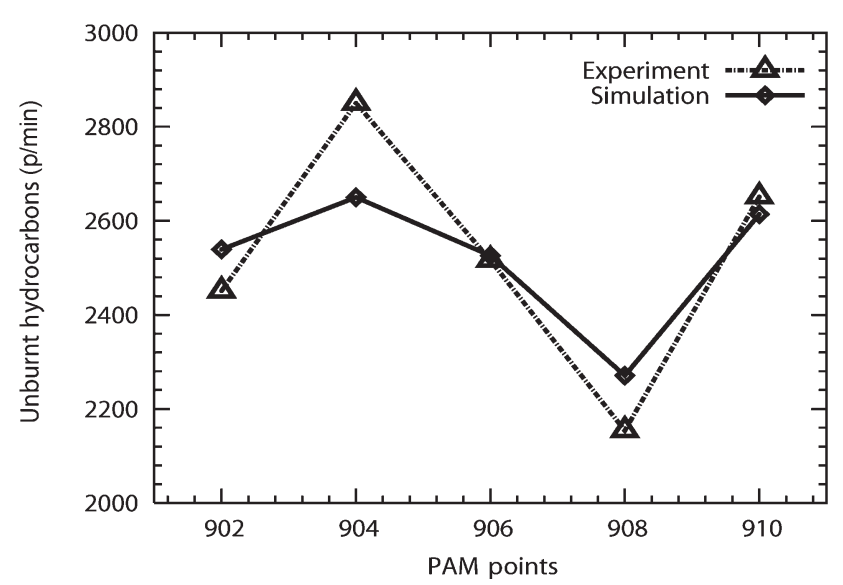

Fig. 11 Trend analysis for UHC formations

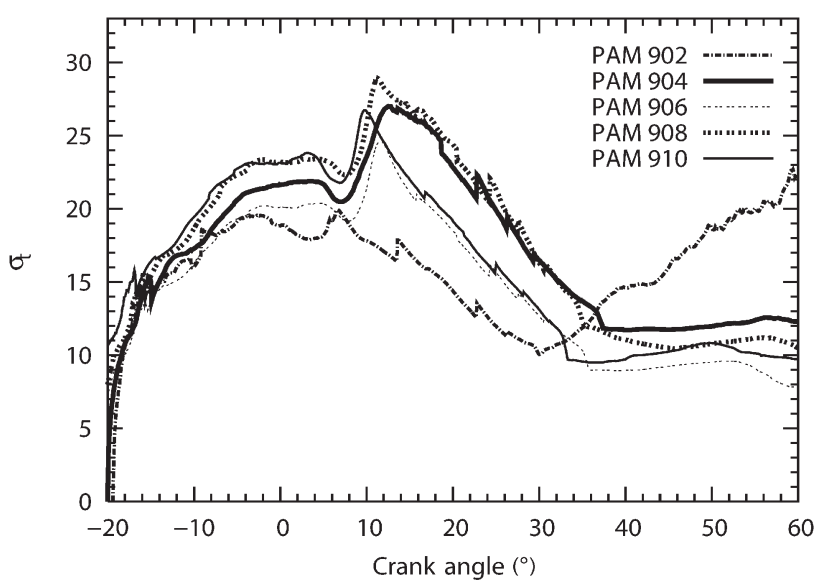

Fig. 12 Turbulent flame surface area ratio $\sigma_{\mathrm{t}}$. Quantities are averaged over the mean turbulent flame front surface

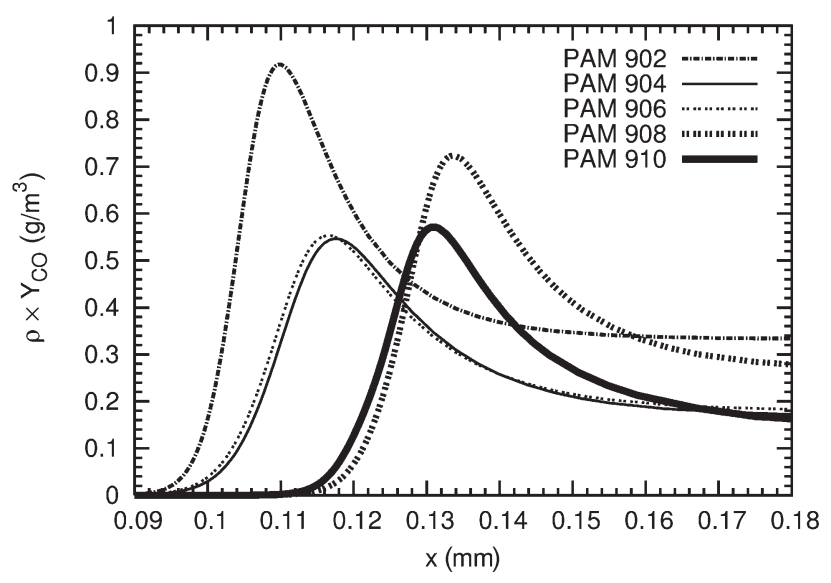

Fig. 13 Carbon monoxide flamelet profiles

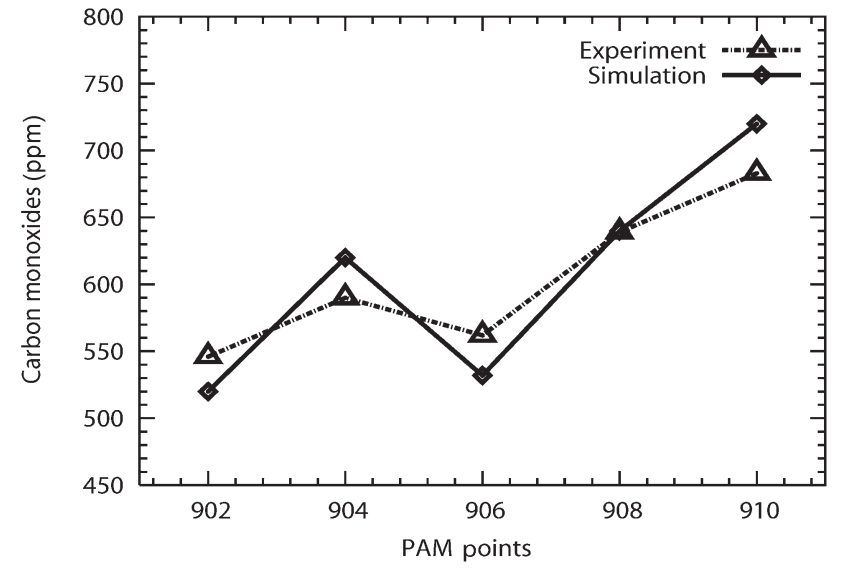

Fig. 14 Trend analysis for COe formation

The G-equation proved successful in predicting pressure traces, characteristic burn points, and apparent heat release rates. In addition, the predicted pollutant formation for nitrogen oxides, UHCs, and CO emissions are in good agreement with the experimental data and the developed phenomenological models are able to capture the evolution trend among all investigated PAMs.

This approach is built on the characteristic turbulent flame brush thickness and therefore it is argued that - contrary to the formulation according to Tan and Reitz [6] - the solution of a transport equation for the variance of $\tilde{G}$ is indispensable, not because the turbulent flame structure could be resolved but because the flame length scale is needed for further flamelet modelling.

However, the last stage of combustion is lacking accuracy and the model failed to predict the 90 per cent burn point. The improvement of the premixed combustion process due to flame-wall interactions will be a subject for study in the near future.

\section{REFERENCES}

1 Peters, N. Turbulent combustion, 2000 (Cambridge University Press).

2 Ewald, J. A level set based flamelet model for the prediction of combustion in homogeneous charge and direct injection spark ignition engines. PhD Thesis, RWTH-Aachen, 2006.

3 Ewald, J. and Peters, N. On unsteady premixed turbulent burning velocity prediction in internal combustion engines. In Proceedings of the Combustion Institute, 2006, pp. 3051-3058. DOI:10.1016/ j.proci.2006.07.119.

4 Herrmann, M. Numerical simulation of turbulent Bunsen flames with a level set flamelet model. Combust. Flame, April 2006, 145, 357-375. 
5 Herrmann, M. Numerical simulation of premixed turbulent combustion based on a level set flamelet model. PhD Thesis, RWTH Aachen, 2001.

6 Tan, Z. and Reitz, R. D. An ignition and combustion model based on the level-set method for spark ignition engine multidimensional modeling. Combust. Flame, 2006, 145, 1-15.

7 Peters, N. The turbulent burning velocity for largescale and small-scale turbulence. J. Fluid Mech., 1999, 384, 107-132.

8 Sethian, J. A. Level set methods and fast marching methods, 1996, 1999 (Cambridge University Press).

9 Tennekes, H. and Lumley, J. L. A first course in turbulence, 1972 (MIT Press, Cambridge, MA).

10 Abdel-Gayed, R. and Bradley, D. Flame propagation along flat plate turbulent boundary layers. Phil. Trans. R. Soc. Lond., A 301, 1981, 1457, 1-25.

11 Damköhler, G. Zeitschr, F. Elektrochemie. Ang. Phys. Chem., 1940, 46(11), 601-626.

12 Peters, N. A spectral closure for premixed turbulent combustion in the flamelet regime. J. Fluid Mech., 1992, 242, 611-629.

13 Launder, B. E. and Spalding, D. B. Computer Methods in Applied Mechanics and Engineering, 1974, 3, 269-89.

14 Seshadri, K. and Peters, N. The inner structure of methane-air flames. Combust. Flame, 1990, 81, 96-118.
15 Müller, U. C., Bollig, M., and Peters, N. Approximation for burning velocities and Markstein numbers for lean hydrocarbon and methanol flames. Combust. Flame, 1997, 108, 349-356.

16 Peters, N. and Williams, F. A. The asymptotic structure of stoichiometric methane-air flames. Combust. Flame, 1987, 68, 185-209.

17 Zeldovich, Y. B. The oxidation of nitrogen in combustion and explosions. Acta Physiochim., USSR 21, 1946, 577.

18 Damköhler, G. Der Einfluss der Turbulenz auf die Flam-mengeschwindigkeit in Gasgemischen. $Z$. Elektrochem, 1940, 46, 601-652.

19 Fenimore, C. P. Studies of fuel-nitrogen in rich flame gases. In Proceedings of the 17th Symposium (International) on Combustion, The Combustion Institute, Pittsburgh, 1979, pp. 661-670.

20 Barths, H. Simulation of diesel engine and gas turbine combustion using multiple flamelets with detailed chemistry. PhD Thesis, RWTH Aachen, 2000.

21 Peters, N. Technische Verbrennung (RWTH Aachen), 2006.

22 Bollig, M. Berechnung laminarer Kohlenwasserstoffflammen im Hinblick auf die Stickoxidbildung in Verbrennungsmotoren. PhD Thesis, RWTH Aachen, 1998. 